\title{
Gastropericardial fistula, uncommon complication for common surgical procedure
}

\author{
Khaled Sherif*, Ali Arvandi, Leigh Ann Jenkins \\ Internal Medicine Department, Texas Tech University Health Science Center, Lubbock, Texas, USA
}

\begin{abstract}
Gastropericardial fistula is an acquired disorder that is associated with a high mortality rate. It is a rare complication of laparoscopic surgery for reflux disease and thoracic procedures. A 74-year-old lady with history of gastroesophageal reflux disease (GERD) and surgically repaired hiatal hernia presented with history of chronic dyspnea and cough. Physical examination was normal apart of a pericardial rub. Laboratory data showed leukocytosis and the chest X-ray showed a large left-sided soft mass. As result of the mass on chest $x$ ray, chest and abdominal CT scan was done and showed large pericardial effusion with hiatal hernia and gastro-pericardial connection. Gastropericardial fistula is an uncommon complication but it has very high mortality rate. Among causes that result in this condition, hiatal hernia and esophagogastric surgery have been the most frequently reported. The occurrence of post-operative gastropericardial fistula is induced by the perihiatal scarring. In addition, recurrence of hiatal hernia or migration of the surgical wrap may also favor occurrence of fistulae. For gastropericardial fistula, contrast media-enhanced traditional examinations are the cornerstone of diagnosis. Abdominal CT may reveal the fistula. Surgical correction is the most effective form of treatment.
\end{abstract}

Keywords: GERD, gastropericardial fistula, hiatus hernia, pericardial effusion

\section{Introduction}

Gastropericardial fistula is an acquired disorder with an abnormal communication between the stomach and the pericardium. It is associated with a high mortality rate [1]. It is a rare complication of laparoscopic surgery for reflux disease and thoracic procedures such as transthoracic esophagostomy and esophageal reconstruction. Nontraumatic fistulae are much less common; asthma, positive pressure ventilation and distal esophageal lesions such as peptic ulceration, malignancy and rupture have been implicated [2]. The hiatal hernia repair can cause substantial life-threatening morbidity like gastropericardial fistula.

Received: January 2016; Accepted after review: March 2016; Published: March 2016.

*Corresponding author: Khaled Sherif, MD, FACP, Internal Medicine Department, Texas Tech University Health Science Center, Lubbock, Texas, USA.

Email: $\underline{k}$ a sherif@hotmail.com

\section{Case report}

A 74-year-old lady with history of GERD and hiatal hernia which was corrected 6 years ago by laparoscopic Nissen fundoplication, presented with dyspnea and cough. She mentioned that her dyspnea started 4 years ago. At first, she had dyspnea on moderate exertion, but in last year it has been progressing and associated with dry cough. Patient said she lost $80 \mathrm{lb}$ in the last year and she was feeling early satiety with intermittent nausea. She had seen many physicians in the past and was treated with different antibiotics for possibilities of community acquired pneumonia. Her dyspnea got worse for 3 days before admission and became even at rest. At time of presentation, the examination showed a pericardial rub and mild epigastric tenderness. The initial laboratory test results included a white blood cell count of $14.2 \mathrm{k} / \mu \mathrm{L}$; hemoglobin, $13.9 \mathrm{~g} / \mathrm{dL}$; platelets, $250 \mathrm{k} / \mu \mathrm{L}$; normal electrolytes; creatinine, $1.1 \mathrm{mg} / \mathrm{dL}$; 
glucose $164 \mathrm{mg} / \mathrm{dL}$; troponin $\mathrm{T},<0.01 \mathrm{ng} / \mathrm{mL}$. mass with cardiomegaly (Figure 1).

The chest $\mathrm{x}$-ray showed a large left-sided soft

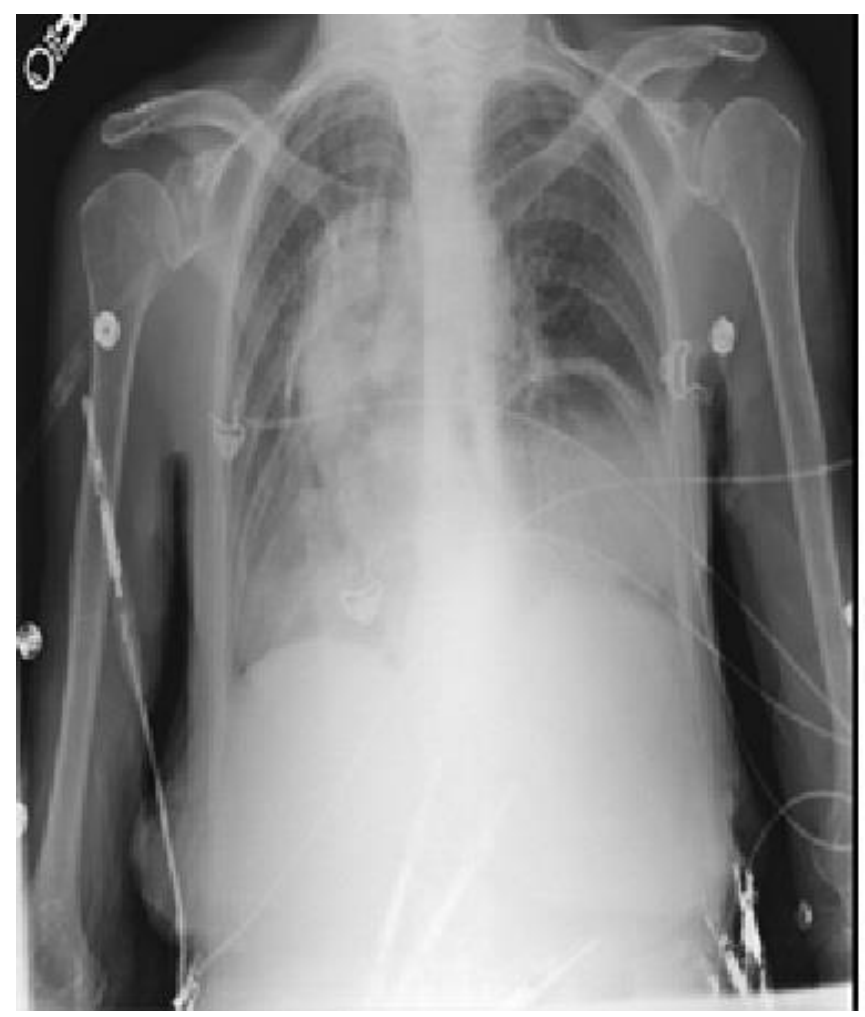

Fig. 1. Large right side soft mass with cardiomegaly

Electrocardiography showed sinus tachycardia with diffuse ST elevation, raising the possibility of acute pericarditis (Figure 2). She had an echocardiogram that showed a large pericardial effusion with physiological tamponade. As result of the mass on chest $x$ ray, non-contrast chest and abdominal CT scan was done. It showed large pericardial effusion with hiatal hernia and gastropericardial connection (Figure 3).

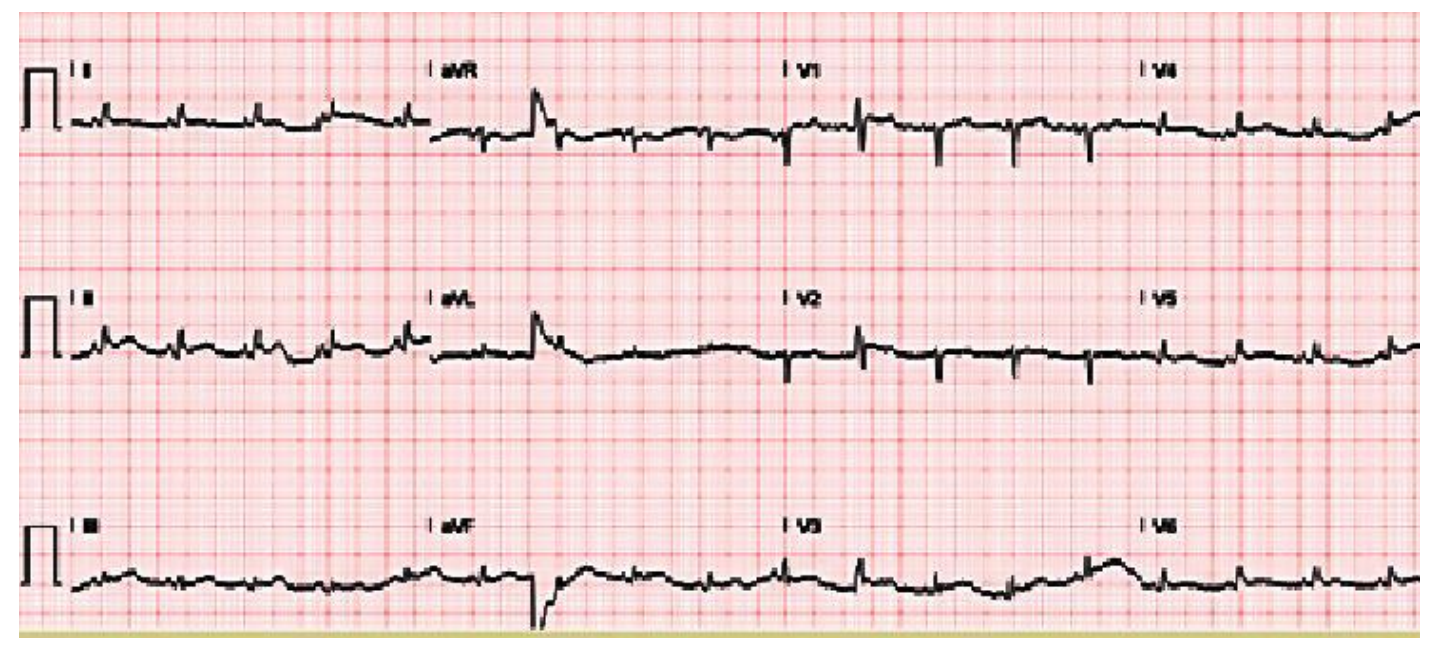

Fig. 2. Sinus tachycardia with diffuse nonspecific $T$ wave changes 


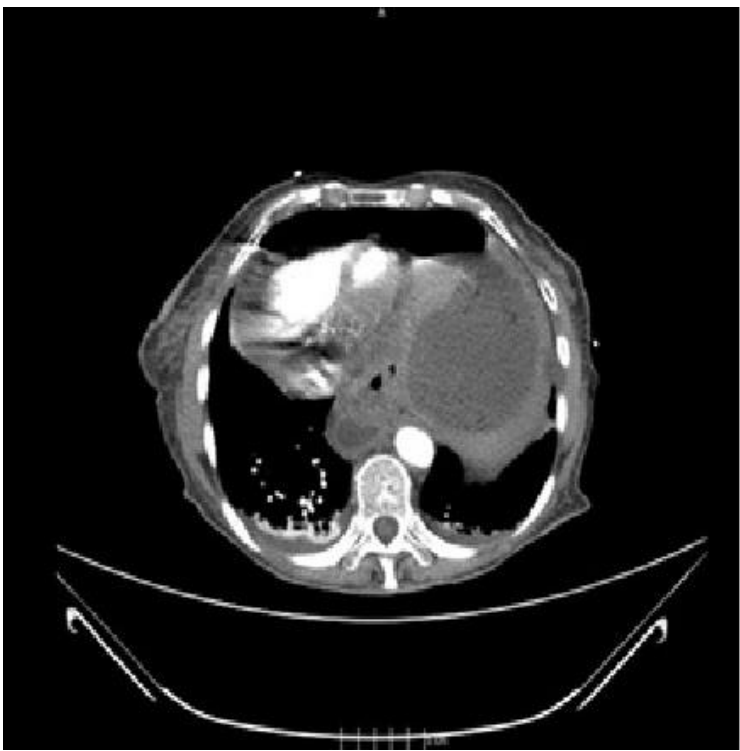

Fig. 3. Large pericardial effusion with hiatal hernia and gastroperocardial connection

After detailed discussion with patient, she underwent a second hiatal hernia repair, left thoracotomy, distal esophagectomy, subtotal gastrectomy, feeding jejunostomy, and pericardial window through thoracoabdominal approach. The procedure was significant for a large gastropericardial fistula, and a large hiatal hernia with gastric and esophageal perforation. The patient tolerated the procedure, and she was transferred to the intensive care unit in critical condition for close monitoring. Four days later, patient had the second step in the planned surgical intervention where she had left thoracotomy with gastroesophageal anastomosis repair. Later, she had a fever episode for which wound, blood and urine cultures were obtained. The culture results showed Candida albicans in wound culture around the chest tube and Enterococcus in the urine, so ampicillin and fluconazole were used for treatment. A follow up esophagram and gastrogram were performed through the nasogastric tube, and showed no gastroesophageal leak (Figure 4). Patient was improving slowly and eventually she was discharged to skilled nursing facility for rehabilitation.

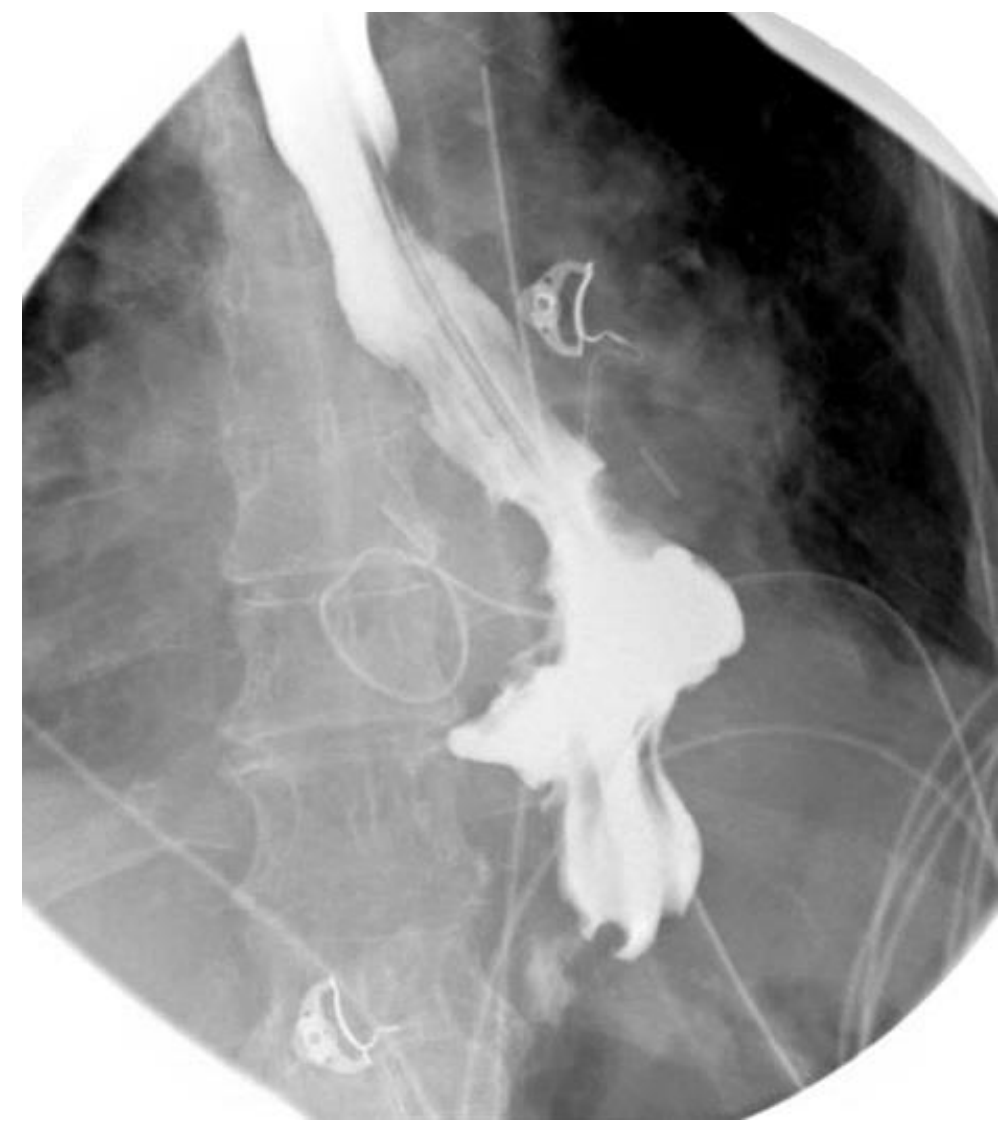

Fig. 4. Post-operative esophagram showed no leak 


\section{Discussion}

First described in 1844 by Bricheteau, gastric or esophageopericardial fistulae are rare entities [3]. Laparoscopic repair of hiatal hernia and GERD is known to be as a safe procedure. More than $90 \%$ of patients have had long-term positive outcome [4]. Gastropericardial fistula is an uncommon complication but it has high mortality rate, estimated around 50\% [5]. Among conditions that result in gastropericardial fistula, peptic ulcer, hiatal hernia and esophagogastric surgery have been the most frequently reported [6]. In our patient, the most likely etiology of gastropericardial fistula was the history of hiatal hernia repair. The postoperative gastropericardial fistula is induced by the perihiatal scarring. In addition, recurrence of hiatal hernia or migration of the surgical wrap may also favor occurrence of fistulae [2]. Clinical features of gastropericardial fistula are variable, ranging from dyspnea and pericardial friction rub to severe chest pain [1]. For gastropericardial fistula, contrast media-enhanced traditional examinations are the cornerstone of diagnosis. Abdominal CT may show the fistula. Our patient did not receive a conventional barium exam as residual barium may produce artifact on subsequent CT. Surgical correction is the

\section{References}

1. Kim WJ, Choi EJ, Oh YW, Kim KT, Kim CW. Gastropericardial fistula-induced pyopneumopericardium after esophagectomy with esophagogastrectomy. Ann Thorac Surg 2011; 91(1):e10-1.

2. Chauhan A, Perry I, Veitch A, Li P, Rattehalli D, Brookes MJ. Gastropericardial fistula: a potential role for conservative treatment. Eur $J$ Gastroenterol Hepatol 2012; 24(11):13411343.

3. Shackelford RT. Hydropneumopericardium. JAMA 1931; 96:187-191.

4. Pessaux P, Arnaud JP, Delattre JF, Meyer C, Baulieux J, Mosnier H. Laparoscopic antireflux most effective form of treatment. Conservative treatment is reserved for those with severe illness and carries a higher mortality rate. In Chauhan and coauthors' report, nasojejunal feeding tube was used along with antibiotics for a patient who was not surgically fit. This will allow adequate nutrition to be administered and reduce the amount of fluid and air going into the fistula, allowing it in theory to close [2].

\section{Conclusion}

The learning point in this case is the delayed diagnosis. Even though there was an abnormal chest $x$-ray along with continuous symptoms, the patient has been treated as having recurrent pneumonia. This case may raise the readers' alertness about the importance of periesophageal disorders that appear in patients who have history of surgical interventions.

\section{Disclosure}

There is nothing needed to be disclosed. All authors read and approved the final manuscript. Every author has contributed in writing the manuscript. surgery: five-year results and beyond in 1340 patients. Arch Surg 2005; 140(10):946-51.

5. Kato T, Mori T, Niibori $K$. A case of gastropericardial fistula of a gastric tube after esophagectomy: a case report and review. World J Emerg Surg 2010; 5:20.

6. Tang CP, Wang YW, Shiau YT, Lee RC, Lan $\mathrm{KH}$, Chao Y. Gastropericardial fistula and Candida albicans pericarditis: a rare complication of gastric adenocarcinoma treated with radiation and chemotherapy. J Chin Med Assoc 2009; 72(7):374-378. 